\title{
Convergence Rate of Riemannian Hamiltonian Monte Carlo and Faster Polytope Volume Computation
}

\author{
Yin Tat Lee \\ University of Washington and Microsoft Research \\ Seattle, WA, USA \\ yintat@uw.edu
}

\author{
Santosh S. Vempala \\ Georgia Tech \\ Atlanta, GA, USA \\ vempala@gatech.edu
}

\begin{abstract}
We give the first rigorous proof of the convergence of Riemannian Hamiltonian Monte Carlo, a general (and practical) method for sampling Gibbs distributions in high dimension. Our analysis shows that the rate of convergence is bounded in terms of natural smoothness parameters of an associated Riemannian manifold. We apply the method to the problems of (1) uniformly sampling a polytope defined by $m$ inequalities in $\mathbb{R}^{n}$ and (2) computing its volume, the latter by extending the Gaussian Cooling algorithm of Cousins and Vempala to the manifold setting. For both problems, we show that the total number of steps needed is $O^{*}\left(m n^{\frac{2}{3}}\right)$, improving the state of the art from $O^{*}\left(m n^{\frac{3}{4}}\right)$ and $O^{*}\left(n^{4}\right)$ steps respectively. A key ingredient of our analysis is a proof of an analog of the KLS conjecture for Gibbs distributions over manifolds.
\end{abstract}

\section{CCS CONCEPTS}

- Theory of computation $\rightarrow$ Probabilistic computation; Random walks and Markov chains; $\bullet$ Mathematics of computing $\rightarrow$ Mathematical analysis;

\section{KEYWORDS}

Polytopes, Hamiltonian Dynamics, Sampling, Volume Computation

\section{ACM Reference Format:}

Yin Tat Lee and Santosh S. Vempala. 2018. Convergence Rate of Riemannian Hamiltonian Monte Carlo and Faster Polytope Volume Computation. In Proceedings of 50th Annual ACM SIGACT Symposium on the Theory of Computing (STOC'18). ACM, New York, NY, USA, 7 pages. https://doi.org/ $10.1145 / 3188745.3188774$

\section{INTRODUCTION}

Hamiltonian dynamics provide an elegant alternative to Newtonian mechanics. The Hamiltonian $H$, which captures jointly the potential and kinetic energy of a particle, is a function of its position and velocity. The following first-order differential equations describe

Permission to make digital or hard copies of all or part of this work for personal or classroom use is granted without fee provided that copies are not made or distributed for profit or commercial advantage and that copies bear this notice and the full citation on the first page. Copyrights for components of this work owned by others than ACM must be honored. Abstracting with credit is permitted. To copy otherwise, or republish, to post on servers or to redistribute to lists, requires prior specific permission and/or a fee. Request permissions from permissions@acm.org.

STOC'18, June 25-29, 2018, Los Angeles, CA, USA

(C) 2018 Association for Computing Machinery.

ACM ISBN 978-1-4503-5559-9/18/06 \$15.00

https://doi.org/10.1145/3188745.3188774 the changes in both.

$$
\begin{aligned}
& \frac{d x}{d t}=\frac{\partial H(x, v)}{\partial v}, \\
& \frac{d v}{d t}=-\frac{\partial H(x, v)}{\partial x} .
\end{aligned}
$$

As we review in Section 2, these equations preserve the Hamiltonian $H$

Riemannian Hamiltonian Monte Carlo [22, 23] (or RHMC [6, 7]) is a Markov Chain Monte Carlo method for sampling from a desired distribution. The target distribution is encoded in the definition of the Hamiltonian. Each step of the method consists of the following: At a current point $x$,

(1) Pick a random velocity $y$ according to a local distribution defined by $x$ (in the simplest setting, this is the standard Gaussian distribution for every $x$ ).

(2) Move along the Hamiltonian curve defined by Hamiltonian dynamics at $(x, y)$ for time (distance) $\delta$.

For a suitable choice of $H$, the marginal distribution of the current point $x$ approaches the desired target distribution. Conceptually, the main advantage of RHMC is that it does not require a Metropolis filter (as in the Metropolis-Hastings method) and its step sizes are therefore not severely limited even in high dimension.

Over the past two decades, RHMC has become very popular in Statistics and Machine Learning, being applied to Bayesian learning, to evaluate expectations and likelihood of large models by sampling from the appropriate Gibbs distribution, etc. It has been reported to significantly outperform other known methods [2,23] and much effort has been made to make each step efficient by the use of numerical methods for solving ODEs.

In spite of all these developments and the remarkable empirical popularity of RHMC, analyzing its rate of convergence and thus rigorously explaining its success has remained an open question.

\subsection{Results}

In this paper, we analyze the mixing rate of Hamiltonian Monte Carlo for a general function $f$ as a Gibbs sampler, i.e., to generate samples from the density proportional to $e^{-f(x)}$. The corresponding Hamiltonian is $H(x, v)=f(x)+\frac{1}{2} \log \left((2 \pi)^{n} \operatorname{det} g(x)\right)+\frac{1}{2} v^{T} g(x)^{-1} v$ for some metric $g$. We show that for $x$ in a compact manifold, the conductance of the Markov chain is bounded in terms of a few parameters of the metric $g$ and the function $f$. The parameters and resulting bounds are given in the full paper. Roughly speaking, the guarantee says that Hamiltonian Monte Carlo mixes in polynomial time for smooth Hamiltonians. We note that these guarantees use only the smoothness and Cheeger constant (expansion) of the function, without any convexity type assumptions. Thus, 
they might provide insight in nonconvex settings where (R)HMC is often applied.

We then focus on logconcave densities in $\mathbb{R}^{n}$, i.e., $f(x)$ is a convex function. This class of functions appears naturally in many contexts and is known to be sampleable in polynomial-time given access to a function value oracle. For logconcave densities, the current fastest sampling algorithms use $n^{4}$ function calls, even for uniform sampling [17, 20], and $n^{2.5}$ oracle calls given a warm start after appropriate rounding (linear transformation) [11]. In the prototypical setting of uniform sampling from a polytope $A x \geq b$, with $m$ inequalities, the general complexity is no better, with each function evaluation taking $O(m n)$ arithmetic operations, for an overall complexity of $n^{4} \cdot m n=m n^{5}$ in the worst case and $n^{2.5} \cdot m n$ after rounding from a warm start. The work of Kannan and Narayanan [9] gives an algorithm of complexity $m n^{2} \cdot m n^{\omega-1}$ from an arbitrary start and $m n \cdot m n^{\omega-1}$ from a warm start (here $\omega$ is the matrix multiplication exponent), which is better than the general case when the number of facets $m$ is not too large. This was recently improved to $m n^{\frac{3}{4}} \cdot m n^{\omega-1}$ from a warm start [12]; the subquadratic complexity for the number of steps is significant since all known general oracle methods cannot below a quadratic number of steps. The leading algorithms and their guarantees are summarized in Table 1.

Table 1: The complexity of uniform polytope sampling from a warm start, where each step of every algorithm uses $\widetilde{O}(n)$ bit of randomness. The entries marked ${ }^{\#}$ are for general convex bodies presented by oracles, while the rest are for polytopes.

\begin{tabular}{|c|l|c|c|}
\hline Year & Algorithm & Steps & Cost per step \\
\hline \hline $1997[8]$ & Ball walk $^{\#}$ & $n^{3}$ & $m n$ \\
\hline $2003[18]$ & Hit-and-run & $n^{3}$ & $m n$ \\
\hline $2009[9]$ & Dikin walk & $m n$ & $m n^{\omega-1}$ \\
\hline $2016[12]$ & Geodesic walk & $m n^{\frac{3}{4}}$ & $m n^{\omega-1}$ \\
\hline $2016[13]$ & Ball walk & $n^{2.5}$ & $m n$ \\
\hline This paper & RHMC & $m n^{\frac{2}{3}}$ & $m n^{\omega-1}$ \\
\hline
\end{tabular}

In this paper, using RHMC, we improve the complexity of sampling polytopes. In fact we do this for a general family of Gibbs distributions, of the form $e^{-\alpha \phi(x)}$ where $\phi(x)$ is a convex function over a polytope. When $\phi(x)$ is the standard logarithmic barrier function and $g(x)$ is its Hessian, we get a sampling method that mixes in only $n^{\frac{1}{6}} m^{\frac{1}{2}}+\frac{n^{\frac{1}{5}} m^{\frac{2}{5}}}{\alpha^{\frac{1}{5}}+m^{-\frac{1}{5}}}+\frac{n^{\frac{2}{3}}}{\alpha+m^{-1}}$ steps from a warm start! When $\alpha=1 / m$, the complexity is $\widetilde{O}\left(m n^{\frac{2}{3}}\right)$ and the resulting distribution is very close to uniform over the polytope.

THEOREM 1.1. Let $\phi$ be the logarithmic barrier for a polytope $M$ with $m$ constraints and $n$ variables. Hamiltonian Monte Carlo applied to the function $f=\exp (-\alpha \phi(x))$ and the metric given by $\nabla^{2} \phi$ with appropriate step size mixes in

$$
\widetilde{O}\left(\frac{n^{\frac{2}{3}}}{\alpha+m^{-1}}+\frac{m^{\frac{1}{3}} n^{\frac{1}{3}}}{\alpha^{\frac{1}{3}}+m^{-\frac{1}{3}}}+m^{\frac{1}{2}} n^{\frac{1}{6}}\right)
$$

steps where each step is the solution of a Hamiltonian ODE.
In recent independent work, Mangoubi and Smith [21] analyze Euclidean HMC in the oracle setting, i.e., assuming an oracle for evaluating $\phi$. Their analysis formally gives a dimensionindependent convergence rate based on certain regularity assumptions, such as strong convexity and smoothness of the Hamiltonian $H$. Unfortunately, these assumptions do not hold for the polytope sampling problem.

An important application of sampling is integration. The known complexity of integration for general logconcave functions is also $n^{4}$ oracle calls [17]. For polytopes, the most natural question is computing its volume. For this problem, the current best complexity is $n^{4} \cdot m n$, where the factor of $O(m n)$ is the complexity of checking membership in a polytope. Thus, even for explicitly specified polytopes, the complexity of estimating the volume from previous work is asymptotically the same as that for a general convex body given by a membership oracle. Here we obtain a volume algorithm with complexity $m n^{\frac{2}{3}} \cdot m n^{\omega-1}$, improving substantially on previous algorithms. The volume algorithm is based using Hamiltonian Monte Carlo for sampling from a sequence of Gibbs distributions over polytopes. We remark that in the case when $m=O(n)^{1}$, the final complexity is $O\left(n^{4.04}\right)$ arithmetic operations, improving by nearly a quadratic factor in the dimension over the previous best complexity of $\tilde{O}\left(n^{6}\right)$ operations for arbitrary polytopes. Table 2 below summarizes progress on the volume problem over the past three decades. Besides improving the complexity of volume computation, each step has typically resulted in new techniques.

Table 2: The complexity of volume estimation, each step uses $\widetilde{O}(n)$ bit of randomness. The current paper applies to general polytopes, and is the first improvement utilizing their structure. The entry marked ${ }^{*}$ is for well-rounded bodies.

\begin{tabular}{|c|l|c|c|}
\hline Year/Authors & New ingredients & Steps & Cost/step \\
\hline \hline 1989/DFK [5] & DFK & $n^{23}$ & $m n$ \\
\hline 1990/LS [15] & Better isoperimetry & $n^{16}$ & $m n$ \\
\hline 1990/L [14] & Ball walk & $n^{10}$ & $m n$ \\
\hline 1991/AK [1] & Logconcave sampling & $n^{10}$ & $m n$ \\
\hline 1990/DF [4] & Better error analysis & $n^{8}$ & $m n$ \\
\hline 1993/LS [16] & Localization lemma & $n^{7}$ & $m n$ \\
\hline 1997/KLS [8] & Speedy walk, isotropy & $n^{5}$ & $m n$ \\
\hline 2003/LV [19] & Annealing, hit-and-run & $n^{4}$ & $m n$ \\
\hline 2015/CV [3]* & Gaussian Cooling & $n^{3}$ & $m n$ \\
\hline This paper & RHMC + Gaussian Cooling & $m n^{\frac{2}{3}}$ & $m n^{\omega-1}$ \\
\hline
\end{tabular}

Theorem 1.2. For any polytope $P=\{x: A x \geq b\}$ with $m$ constraints and $n$ variables, and any $\varepsilon>0$, the Hamiltonian volume algorithm estimates the volume of $P$ to within $1 \pm \varepsilon$ multiplicative factor using $\widetilde{O}\left(m n^{\frac{2}{3}} \varepsilon^{-2}\right)$ steps where each step consists of solving a

${ }^{1}$ We suspect that the LS barrier [10] might be used to get a faster algorithm even in the regime $m$ is sub-exponential. However, our proof requires a delicate estimate of the fourth derivative of the barrier functions. Therefore, such a result either requires a new proof or an unpleasantly long version of the current proof. 
first-order $O D E$ and takes time $\widetilde{O}\left(m n^{\omega-1} L^{O(1)} \log O(1) \frac{1}{\varepsilon}\right)$ and $L$ is the bit complexity ${ }^{2}$ of the polytope.

A key ingredient in the analysis of RHMC is a new isoperimetric inequality for Gibbs distributions over manifolds (Lemma 3.5). This inequality can be seen as an evidence of a manifold version of the KLS hyperplane conjecture. For the family of Gibbs distributions induced by convex functions with convex Hessians, the expansion is within a constant factor of that of a hyperplane cut. This result might be of independent interest.

\subsection{Approach and Contributions}

Traditional methods to sample from distributions in $\mathbb{R}^{n}$ are based on random walks that take straight line steps (grid walk, ball walk, hit-and-run). While this leads to polynomial-time convergence for logconcave distributions, the length of each step has to be small due to boundary effects, and a Metropolis filter (rejection sampling) has to be applied to ensure the limiting distribution is the desired one. These walks cannot afford a step of length greater than $\delta=O\left(\frac{1}{\sqrt{n}}\right)$ for a distribution in isotropic position, and take a quadratic number of steps even for the hypercube. The Dikin walk for polytopes [9], which explicitly takes into account the boundary of polytope at each step, has a varying step size, but still runs into similar issues and the bound on its convergence rate is $O(m n)$ for a polytope with $m$ facets.

In a recent paper [12], we introduced the geodesic walk. Rather than using straight lines in Euclidean space, each step of the walk is along a geodesic (locally shortest path) of a Riemannian metric. More precisely, each step first makes a deterministic move depending on the current point (drift), then moves along a geodesic in a random initial direction and finally uses a Metropolis filter. Each step can be computed by solving a first-order ODE. Due to the combination of drift and geodesic, the local 1-step distributions are smoother than that of the Dikin walk and larger steps can be taken while keeping a bounded rejection probability for the filter. For sampling polytopes, the manifold/metric defined by the standard log barrier gives a convergence rate of $m n^{\frac{3}{4}}$, going below the quadratic (or higher) bound of all previous sampling methods.

One major difficulty with geodesic walk is ensuring the stationary distribution is uniform. For high dimensional problems, this necessitates taking a sufficiently small step size and then rejecting some samples according to the desired transition probabilities according to Metropolis filter. Unfortunately, computing these transition probabilities can be very expensive. For the geodesic walk, it entails solving an $n \times n$ size matrix ODE.

Hamiltonian Monte Carlo bears some similarity to the geodesic walk - each step is a random (non-linear) curve. But the Hamiltonian-preserving nature of the process obviates the most expensive ingredient, Metropolis filter. Due to this, the step size can be made longer, and as a result we obtain a faster sampling algorithm for polytopes that mixes in $m n^{\frac{2}{3}}$ steps (the per-step complexity remains essentially the same, needing the solution of an ODE).

${ }^{2} L=\log \left(m+d_{\max }+\|b\|_{\infty}\right)$ where $d_{\max }$ is the largest absolute value of the determinant of a square sub-matrix of $A$.
To get a faster algorithm for volume computation, we extend the analysis to a general family of Gibbs distributions, including $f(x)=e^{-\alpha \phi(x)}$ where $\phi(x)$ is the standard log-barrier and $\alpha>0$. We show that the smoothness we need for the sampling corresponding to a variant of self-concordance. Furthermore, we establish an isoperimetric inequality for this class of functions. This can be viewed as an extension of the KLS hyperplane conjecture from Euclidean to Riemannian metrics (the analogous case in Euclidean space to what we prove here is the isoperimetry of the Gaussian density function multiplied by any logconcave function, a case for which the KLS conjecture holds). The mixing rate for this family of functions is sublinear for $\alpha=\Omega(1)$.

Finally, we study the Gaussian Cooling schedule of [3]. We show that in the manifold setting, the Gaussian distribution $e^{-\|x\|^{2} / 2}$ can be replaced by $e^{-\alpha \phi(x)}$. Moreover, the speed of Gaussian Cooling depends on the "thin-shell" constant of the manifold and the classical self-concordance of $\phi$.

Combining all of these ideas, we obtain a faster algorithm for polytope volume computation. The resulting complexity of polytope volume computation is the same as that of sampling uniformly from a warm start: $m n^{\frac{2}{3}}$ steps. To illustrate the improvement, for polytopes with $m=O(n)$ facets, the new bound is $n^{\frac{5}{3}}$ while the previous best bound was $n^{4}$.

The full version of this paper appears on the Arxiv with id 1710.06261 .

\subsection{Practicality}

From the experiments, the ball walk/hit-and-run seem to mix in $n^{2}$ steps, the geodesic walk seems to mix in sublinear number of steps (due to the Metropolis filter bottleneck) and RHMC seems to mix in only polylogarithmic number of steps. One advantage of RHMC compared to the geodesic walk is that it does not require the expensive Metropolis filter that involves solving $n \times n$ matrix ODEs. In the future, we plan to do an empirical comparison study of different sampling algorithms. We are hopeful that using RHMC we might finally be able to sample from polytopes in millions of dimensions after more than three decades of research on this topic!

\section{BASICS OF HAMILTONIAN MONTE CARLO}

In this section, we define the Hamiltonian Monte Carlo method for sampling from a general distribution $e^{-H(x, y)}$. Hamiltonian Monte Carlo uses curves instead of straight lines and this makes the walk time-reversible even if the target distribution is not uniform, with no need for a rejection sampling step. In contrast, classical approaches such as the ball walk require an explicit rejection step to converge to a desired stationary distribution.

Definition 2.1. Given a continuous, twice-differentiable function $H: \mathcal{M} \times \mathbb{R}^{n} \subset \mathbb{R}^{n} \times \mathbb{R}^{n} \rightarrow \mathbb{R}$ (called the Hamiltonian, which often corresponds to the total energy of a system) where $\mathcal{M}$ is the $x$ domain of $H$, we say $(x(t), y(t))$ follows a Hamiltonian curve if it satisfies the Hamiltonian equations 


$$
\begin{aligned}
& \frac{d x}{d t}=\frac{\partial H(x, y)}{\partial y}, \\
& \frac{d y}{d t}=-\frac{\partial H(x, y)}{\partial x} .
\end{aligned}
$$

We define the map $T_{\delta}(x, y) \stackrel{\text { def }}{=}(x(\delta), y(\delta))$ where the $(x(t), y(t))$ follows the Hamiltonian curve with the initial condition $(x(0), y(0))=$ $(x, y)$.

Hamiltonian Monte Carlo is the result of a sequence of randomly generated Hamiltonian curves.

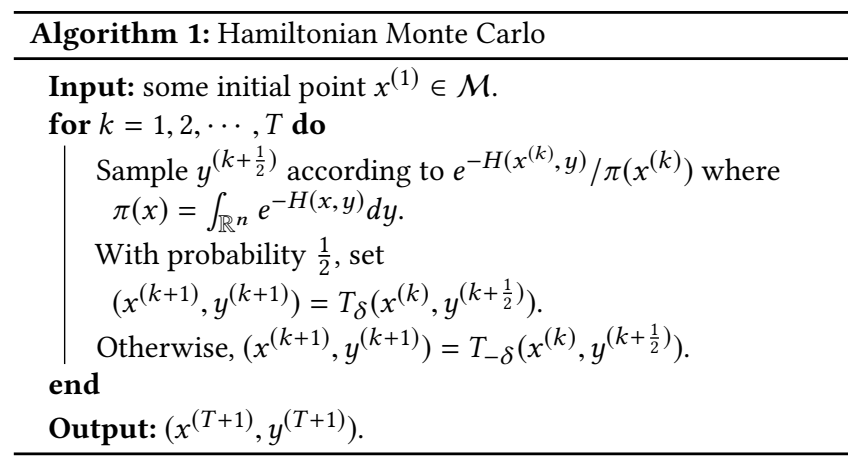

Here we two key properties of Hamiltonian Monte Carlo method.

Lemma 2.2 (Energy Conservation). For any Hamiltonian curve $(x(t), y(t))$, we have that

$$
\frac{d}{d t} H(x(t), y(t))=0 .
$$

Lemma 2.3 (Measure Preservation). For any $t \geq 0$, we have that

$$
\operatorname{det}\left(D T_{t}(x, y)\right)=1
$$

where $D T_{t}(x, y)$ is the Jacobian of the map $T_{t}$ at the point $(x, y)$.

Using the previous two properties, one can show that Hamiltonian Monte Carlo indeed converges to the desired distribution.

Lemma 2.4 (Time Reversibility). Let $p_{x}\left(x^{\prime}\right)$ denote the probability density of one step of the Hamiltonian Monte Carlo starting at $x$. We have that

$$
\pi(x) p_{x}\left(x^{\prime}\right)=\pi\left(x^{\prime}\right) p_{x^{\prime}}(x)
$$

for almost everywhere in $x$ and $x^{\prime}$ where $\pi(x)=\int_{\mathbb{R}^{n}} e^{-H(x, y)} d y$.

The main challenge in analyzing Hamiltonian Monte Carlo is to bound its mixing time.

\subsection{Hamiltonian Monte Carlo on Riemannian Manifolds}

Suppose we want to sample from the distribution $e^{-f(x)}$. We define the following energy function $H$ :

$$
H(x, v) \stackrel{\text { def }}{=} f(x)+\frac{1}{2} \log \left((2 \pi)^{n} \operatorname{det} g(x)\right)+\frac{1}{2} v^{T} g(x)^{-1} v .
$$

where $g$ is some metric (positive definite matrix) at $x$. One can view $x$ as the location and $v$ as the velocity. The following lemma shows that the first variable $x(t)$ in the Hamiltonian curve satisfies a second-order differential equation. When we view the domain $\mathcal{M}$ as a manifold, this equation is simply $D_{t} \frac{d x}{d t}=\mu(x)$, namely, $x$ acts like a particle under the force field $\mu$.

Lemma 2.5. In Euclidean coordinates, The Hamiltonian equation for (2.2) can be rewritten as

$$
\begin{aligned}
& D_{t} \frac{d x}{d t}=\mu(x), \\
& \frac{d x}{d t}(0) \sim N\left(0, g(x)^{-1}\right)
\end{aligned}
$$

where $\mu(x)=-g(x)^{-1} \nabla f(x)-\frac{1}{2} g(x)^{-1} \operatorname{Tr}\left[g(x)^{-1} D g(x)\right]$ and $D_{t}$ is the Levi-Civita connection on the manifold $\mathcal{M}$ with metric $g$.

Motivated by this, we define the Hamiltonian map as the first component of the Hamiltonian dynamics operator $T$ defined earlier. For the reader familiar with Riemannian geometry, this is similar to the exponential map.

Definition 2.6. Let $\operatorname{Ham}_{x, \delta}\left(v_{x}\right)=\gamma(\delta)$ where $\gamma(t)$ be the solution of the Hamiltonian equation $D_{t} \frac{d \gamma}{d t}=\mu$ with initial conditions $\gamma(0)=x$ and $\gamma^{\prime}(0)=v_{x}$. We also denote $\operatorname{Ham}_{x, 1}\left(v_{x}\right)$ by $\operatorname{Ham}_{x}\left(v_{x}\right)$.

We now give two examples of Hamiltonian Monte Carlo. Example. When $g(x)=I$ (namely, the manifold is the Euclidean space) and the target distribution is a general logconcave distribution $\exp (-f(x))$, the Hamiltonian curve acts like stochastic gradient descent for the function $f$ with each random perturbation drawn from a standard Gaussian.

$$
D_{t} \frac{d x}{d t}=-\nabla f(x)
$$

When $g(x)=\nabla^{2} f(x)$, the Hamiltonian curve acts like a stochastic Newton curve for the function $f+\psi$ :

$$
D_{t} \frac{d x}{d t}=-\left(\nabla^{2} f(x)\right)^{-1} \nabla(f(x)+\psi(x))
$$

where the volumetric function $\psi(x)=\log \operatorname{det} \nabla^{2} f(x)$.

\section{CONVERGENCE OF RIEMANNIAN HAMILTONIAN MONTE CARLO}

Hamiltonian Monte Carlo is a Markov chain on a manifold whose stationary stationary distribution has density $q(x)$ proportional to $\exp (-f(x))$. We will bound the conductance of this Markov chain and thereby its mixing time to converge to the stationary distribution. Bounding conductance involves showing (a) the induced metric on the state space satisfies a strong isoperimetric inequality and (b) two points that are close in metric distance are also close in probabilistic distance, i.e., the one-step distributions from two nearby points have large overlap.

Given a Markov chain with state space $\mathcal{M}$ with stationary distribution $q$ and next step distribution $p_{u}(\cdot)$ for any $u \in \mathcal{M}$. One can bound the mixing time, i.e., the rate of convergence to its stationary distribution, of the Markov chain via the isoperimetry of the metric space. Using results by Lovï œesz and Simonovits, we have the following result for bounding mixing time of general geometric random walk. 
Lemma 3.1. Given a metric space $\mathcal{M}$ and a time-reversible Markov chain $p$ on $\mathcal{M}$ with stationary distribution q. Suppose that there exist $r>0$ and $\psi>0$ such that

(1) For any $x, y \in \mathcal{M}$ with $d(x, z)<r$, we have that $d_{T V}\left(p_{x}, p_{y}\right) \leq$ 0.9 .

(2) For any $S \subset \mathcal{M}$, we have that

$$
\int_{0<d(S, x) \leq r} q(x) d x \geq r \psi \min \{q(S), q(\mathcal{M} \backslash S)\} .
$$

Let $q_{t}$ be the distribution of the current point after $t$ steps of a Markov chain with stationary distribution $q$ starting from initial distribution $q_{0}$. For any $\varepsilon>0$,

$$
d_{T V}\left(q_{t}, q\right) \leq \varepsilon+\sqrt{\frac{1}{\varepsilon} \mathbb{E}_{X \sim q_{0}} \frac{d q_{0}(x)}{d q(x)}}\left(1-\Omega\left(r^{2} \psi^{2}\right)\right)^{t} .
$$

To compute $d_{T V}\left(p_{x}, p_{y}\right)$, we first derive a formula for the transition probability in Euclidean coordinates.

Lemma 3.2. For any $x \in \mathcal{M} \subset \mathbb{R}^{n}$ and $s>0$, the probability density of the 1-step distribution from $x$ is given by $p_{x}(y)$ equal to

$$
\sum_{v_{x}: \operatorname{Ham}_{x, \delta}\left(v_{x}\right)=y} \frac{1}{\left|\operatorname{det}\left(D \operatorname{Ham}_{x, \delta}\left(v_{x}\right)\right)\right|} \sqrt{\frac{\operatorname{det}(g(y))}{(2 \pi)^{n}}} \exp \left(-\frac{1}{2}\left\|v_{x}\right\|_{x}^{2}\right)
$$

where $\operatorname{Ham}_{x, \delta}\left(v_{x}\right)$ is the Jacobian of the Hamiltonian map $\operatorname{Ham}_{x, \delta}$.

In general, there can be infinitely many $v$ such that $\operatorname{Ham}_{x, \delta}(v)=$ $y$. For example, Hamiltonian dynamic on Euclidean space is just rotation and $v$ indicates the angle of the rotation. Therefore, there are either 0 or infinitely many rotations that maps $x$ to $y$. However, there are only one term with large probability and hence one can ignore the summation for the intuition.

To analyze this formula, we introduce the concept of variations of Hamiltonian curves and use it to bound $\left|\operatorname{det}\left(D \operatorname{Ham}_{x, \delta}\left(v_{x}\right)\right)\right|^{-1}$. We can show that $p_{x}(y)$ is in fact close to

$$
\tilde{p}_{x}(y)=\sum_{v_{x}: \operatorname{Ham}_{x, \delta}\left(v_{x}\right)=y} \frac{1}{\delta^{n}} \cdot \sqrt{\frac{\operatorname{det}(g(y))}{(2 \pi)^{n}}} \exp \left(-\frac{1}{2}\left\|v_{x}\right\|_{x}^{2}\right) .
$$

To compare $p_{x}(y)$ with $p_{z}(y)$, we need to relate $v_{x}$ and $v_{z}$ that map $x$ and $z$ to $y$ respectively. In the full paper, we show that if $x$ and $z$ are close enough, for every $v_{x}$, there is a unique $v_{z}$ such that $v_{x}$ is close to $v_{z}$ and that $\operatorname{Ham}_{z, \delta}\left(v_{z}\right)=\operatorname{Ham}_{x, \delta}\left(v_{x}\right)$. Combining these facts and some tedious calculation involving log barrier functions, we show the following estimate.

Lemma 3.3. Let $\mathcal{M} \subset \mathbb{R}^{n}$ be the Hessian manifold on induced by the log barrier function $\phi(x)=\sum_{i=1}^{m}-\ln \left(a_{i}^{T} x-b_{i}\right)$, namely, the metric $g_{i j}$ at $x$ is given by $\nabla^{2} \phi(x)_{i j}$. Consider the Hamiltonian walk on $\mathcal{M}$ with the target distribution $\exp (-\alpha \cdot \phi(x))$. Assume that the step size is

$$
\delta \leq c \cdot \min \left(n^{-\frac{1}{3}}, \alpha^{-\frac{1}{3}} m^{-\frac{1}{6}} n^{-\frac{1}{6}}, \alpha^{-\frac{1}{2}} m^{-\frac{1}{4}} n^{-\frac{1}{12}}\right),
$$

Then the one-step Hamiltonian walk distributions $p_{x}, p_{z}$ from $x, z$ satisfy

$$
d_{\mathrm{TV}}\left(p_{x}, p_{z}\right)=O\left(\frac{1}{\delta}\right) d(x, z)+\frac{1}{25}
$$

For the second condition, we derive a general isoperimetry bound, assuming that the manifold is defined by the Hessian of a convex function, and that the directional fourth directive is nonnegative, a property satisfied, e.g., by the standard logarithmic barrier.

LEMmA 3.4. Let $\phi:[a, b] \rightarrow \mathbb{R}$ be a convex function such that $\phi^{\prime \prime}$ is also convex and $f(x)$ be the logconcave density proportional to $e^{-\phi(x)}$. Then, we have that

$$
\operatorname{Var}_{X \sim f(x)} X \leq \frac{O(1)}{\min _{x \in[a, b]} \phi^{\prime \prime}(x)} .
$$

This generalizes to higher dimension with no dependence on the dimension using localization.

Lemma 3.5. Let $\phi: K \subset \mathbb{R}^{n} \rightarrow \mathbb{R}$ be a convex function defined over a convex body K such that $D^{4} \phi(x)[h, h, h, h] \geq 0$ for all $x \in K$ and $h \in$ $\mathbb{R}^{n}$. Given any partition $S_{1}, S_{2}, S_{3}$ of $K$ with $d=\min _{x \in S_{1}, y \in S_{2}} d(x, y)$, i.e., the minimum distance between $S_{1}$ and $S_{2}$ in the Riemannian metric induced by $\phi$. For any $\alpha>0$, we have that

$$
\frac{\int_{S_{3}} e^{-\alpha \phi(x)} d x}{\min \left\{\int_{S_{1}} e^{-\alpha \phi(x)} d x, \int_{S_{2}} e^{-\alpha \phi(x)} d x\right\}}=\Omega(\sqrt{\alpha} \cdot d) .
$$

For the case small $\alpha \geq 0$, the above bound is not sufficient for getting the bound of the mixing time. We note that for the case $\phi(x)$ is the log barrier function, [12] showed that

$$
\frac{\int_{S_{3}} e^{-\alpha \phi(x)} d x}{\min \left\{\int_{S_{1}} e^{-\alpha \phi(x)} d x, \int_{S_{2}} e^{-\alpha \phi(x)} d x\right\}}=\Omega(\sqrt{1 / m} \cdot d) .
$$

Theorem 1.1 now follows from this, Lemma 3.3, Lemma 3.5 and Lemma 3.1.

\section{POLYTOPE VOLUME COMPUTATION: GAUSSIAN COOLING ON MANIFOLDS}

The volume algorithm is essentially the Gaussian cooling algorithm introduced in [3]. Here we apply it to a sequence of Gibbs distributions rather than a sequence of Gaussians. More precisely, for a convex body $K$ and a convex barrier function $\phi: K \rightarrow \mathbb{R}$, we define

$$
f\left(\sigma^{2}, x\right)= \begin{cases}\exp \left(-\sigma^{-2} \phi(x)\right) & \text { if } x \in K \\ 0 & \text { otherwise }\end{cases}
$$

and

$$
F\left(\sigma^{2}\right)=\int_{\mathbb{R}^{n}} f\left(\sigma^{2}, x\right) d x
$$

where $x^{*}$ is the minimizer of $\phi$ (the center of $K$ ). Let $\mu_{i}$ be the probability distribution proportional to $f\left(\sigma_{i}^{2}, x\right)$ where $\sigma_{i}$ is the tempature of the Gibbs distribution to be fixed. The algorithm estimates each ratio in the following telescoping product:

$$
e^{-\sigma^{-2} \phi\left(x^{*}\right)} \operatorname{vol}(K) \approx F\left(\sigma_{k}\right)=F\left(\sigma_{0}\right) \prod_{i=1}^{k} \frac{F\left(\sigma_{i+1}^{2}\right)}{F\left(\sigma_{i}^{2}\right)}
$$

for some large enough $\sigma_{k}$.

Let $x$ be a random sample point from $\mu_{i}$ and let

$$
Y_{x}=f\left(\sigma_{i+1}^{2}, x\right) / f\left(\sigma_{i}^{2}, x\right) .
$$


Then,

$$
\mathbb{E}_{x \sim \mu_{i}}\left(Y_{x}\right)=\frac{F\left(\sigma_{i+1}^{2}\right)}{F\left(\sigma_{i}^{2}\right)} .
$$

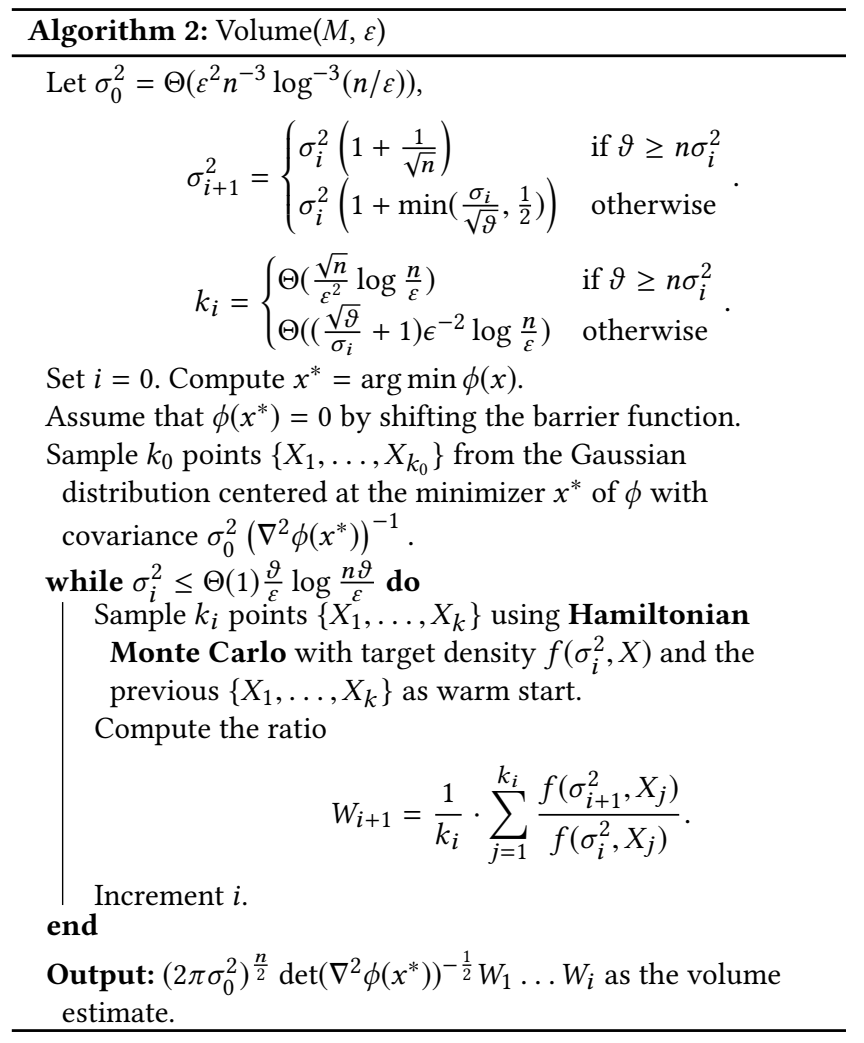

We separate the proof into two parts. In the first part, we estimate how small $\sigma_{0}$ we start with should be and how large $\sigma_{k}$ we end with should be.

LEMma 4.1 (LARGE AND SMALL $\sigma^{2}$ ). Let $\phi$ be a $\vartheta$-self concordant barrier function for $K$. If $\sigma n^{\frac{3}{2}} \log ^{\frac{3}{2}} \frac{1}{\sigma} \leq 1$ and $\sigma n^{\frac{1}{2}} \log ^{\frac{1}{2}} \frac{1}{\sigma} \leq 1$, then we have that

$$
\begin{aligned}
& \left|\ln F(\sigma)-\ln \left(e^{-\sigma^{-2} \phi\left(x^{*}\right)}\left(2 \pi \sigma^{2}\right)^{\frac{n}{2}} \operatorname{det}\left(\nabla^{2} \phi\left(x^{*}\right)\right)^{-\frac{1}{2}}\right)\right| \\
& \leq O\left(\sigma n^{\frac{3}{2}} \log ^{\frac{3}{2}} \frac{1}{\sigma}\right) .
\end{aligned}
$$

If $\sigma^{2} \geq \vartheta$, then

$$
\left|\ln F(\sigma)-\ln \left(e^{-\sigma^{-2} \phi\left(x^{*}\right)} \operatorname{vol}(K)\right)\right| \leq O\left(\sigma^{-2} \vartheta \ln \left(\sigma^{2} n / \vartheta\right)\right) .
$$

In the second part, we estimate the variance of the estimator $Y_{x}$. Our goal is to estimate $\mathbb{E}_{x \sim \mu_{i}}\left(Y_{x}\right)$ within a target relative error. The algorithm estimates the quantity $\mathbb{E}_{x \sim \mu_{i}}\left(Y_{x}\right)$ by taking random sample points $x_{1}, \ldots, x_{k}$ and computing the empirical estimate for $\mathbb{E}_{x \sim \mu_{i}}\left(Y_{x}\right)$ from the corresponding $Y_{x_{1}}, \ldots, Y_{x_{k}}$. The variance of $Y_{x_{i}}$ divided by its expectation squared will give a bound on how many independent samples $x_{i}$ are needed to estimate $\mathbb{E}_{x \sim \mu_{i}}\left(Y_{x}\right)$ within the target accuracy. We have

$$
\mathbb{E}_{x \sim \mu_{i}}\left(Y_{x}^{2}\right)=\frac{\int_{K} \exp \left(\frac{\phi(x)}{\sigma_{i}^{2}}-\frac{2 \phi(x)}{\sigma_{i+1}^{2}}\right) d x}{\int_{K} \exp \left(-\frac{\phi(x)}{\sigma_{i}^{2}}\right) d x}=\frac{F\left(\frac{\sigma_{i+1}^{2} \sigma_{i}^{2}}{2 \sigma_{i}^{2}-\sigma_{i+1}^{2}}\right)}{F\left(\sigma_{i}^{2}\right)}
$$

and

$$
\frac{\mathbb{E}_{x \sim \mu_{i}}\left(Y_{x}^{2}\right)}{\mathbb{E}_{x \sim \mu_{i}}\left(Y_{x}\right)^{2}}=\frac{F\left(\sigma_{i}^{2}\right) F\left(\frac{\sigma_{i+1}^{2} \sigma_{i}^{2}}{2 \sigma_{i}^{2}-\sigma_{i+1}^{2}}\right)}{F\left(\sigma_{i+1}^{2}\right)^{2}}
$$

If we let $\sigma^{2}=\sigma_{i+1}^{2}$ and $\sigma_{i}^{2}=\sigma^{2} /(1+r)$, then we can further simplify as

$$
\frac{\mathbb{E}_{x \sim \mu_{i}}\left(Y_{x}^{2}\right)}{\mathbb{E}_{x \sim \mu_{i}}\left(Y_{x}\right)^{2}}=\frac{F\left(\frac{\sigma^{2}}{1+r}\right) F\left(\frac{\sigma^{2}}{1-r}\right)}{F\left(\sigma^{2}\right)^{2}} .
$$

LemmA 4.2. For any $1>r \geq 0$, we have that

$$
\ln \left(\frac{F\left(\frac{\sigma^{2}}{1+r}\right) F\left(\frac{\sigma^{2}}{1-r}\right)}{F\left(\sigma^{2}\right)^{2}}\right)=\frac{1}{\sigma^{4}} \int_{0}^{r} \int_{1-t}^{1+t} \operatorname{Var}_{x \sim \mu_{s}} \phi(x) d s d t
$$

where $\mu_{s}$ be the probability distribution proportional to $f\left(\frac{\sigma^{2}}{s}, x\right)$.

Next we bound $\operatorname{Var}_{x \sim \mu_{s}} \phi(x)$. This can be viewed as a manifold version of the thin shell or variance hypothesis estimate.

Lemma 4.3 (Thin Shell estimates). Let $\phi$ be a $\vartheta$-self concordant barrier function for $K$. Then, we have that

$$
\operatorname{Var}_{x \sim \mu_{s}} \phi(x)=O\left(\frac{\sigma^{2}}{s} \vartheta\right) .
$$

Now we are ready to state the key lemma. This lemma explains why we increase the temperature $\sigma^{2}$ by a $(1+r)$ factor with $r=$ $\max \left(\frac{\sigma}{\sqrt{\vartheta}}, \frac{1}{\sqrt{n}}\right)$.

LEMma 4.4. Let $\phi$ be a $\vartheta$-self concordant barrier function for $K$. For any $\frac{1}{2}>r \geq 0$, we have that

$$
\frac{\mathbb{E}_{x \sim \mu_{i}}\left(Y_{x}^{2}\right)}{\mathbb{E}_{x \sim \mu_{i}}\left(Y_{x}\right)^{2}}=O\left(r^{2}\right) \min \left(\frac{\vartheta}{\sigma_{i}^{2}}, n\right) .
$$

\section{ACKNOWLEDGEMENT}

We thank Ben Cousins for helpful discussions. This work was supported in part by NSF awards CCF-1563838, CCF-1717349, CCF1740551 and NSF 1749609. Part of this work was done while visiting the Simons Institute for the Theory of Computing, UC Berkeley.

\section{REFERENCES}

[1] D. Applegate and R. Kannan. 1991. Sampling and integration of near log-concave functions. In STOC. 156-163.

[2] M. Betancourt. 2017. A Conceptual Introduction to Hamiltonian Monte Carlo. ArXiv e-prints (Jan. 2017). arXiv:stat.ME/1701.02434

[3] B. Cousins and S. Vempala. 2015. Bypassing KLS: Gaussian cooling and an $O^{*}\left(n^{3}\right)$ volume algorithm. In STOC. 539-548.

[4] M.E. Dyer and A. M. Frieze. 1991. Computing the volume of a convex body: a case where randomness provably helps. In Proc. of AMS Symposium on Probabilistic Combinatorics and Its Applications. 123-170.

[5] M. E. Dyer, A. M. Frieze, and R. Kannan. 1989. A Random Polynomial Time Algorithm for Approximating the Volume of Convex Bodies. In STOC. 375-381. 
[6] Mark Girolami and Ben Calderhead. 2011. Riemann manifold Langevin and Hamiltonian Monte Carlo methods. Fournal of the Royal Statistical Society: Series B (Statistical Methodology) 73, 2 (2011), 123-214. https://doi.org/10.1111/j.1467-9868. 2010.00765.x

[7] M. Girolami, B. Calderhead, and S. A. Chin. 2009. Riemannian Manifold Hamiltonian Monte Carlo. ArXiv e-prints (July 2009). arXiv:stat.CO/0907.1100

[8] R. Kannan, L. Lovász, and M. Simonovits. 1997. Random walks and an $O^{*}\left(n^{5}\right)$ volume algorithm for convex bodies. Random Structures and Algorithms 11 (1997), $1-50$.

[9] R. Kannan and H. Narayanan. 2009. Random walks on polytopes and an affine interior point method for linear programming. In STOC. 561-570.

[10] Yin Tat Lee and Aaron Sidford. 2014. Path finding methods for linear program ming: Solving linear programs in o (vrank) iterations and faster algorithms for maximum flow. In Foundations of Computer Science (FOCS), 2014 IEEE $55^{\text {th }}$ Annual Symposium on. IEEE, 424-433.

[11] Yin Tat Lee and Santosh Srinivas Vempala. 2016. Eldan's Stochastic Localization and the KLS Hyperplane Conjecture: An Improved Lower Bound for Expansion. CoRR abs/1612.01507 (2016). http://arxiv.org/abs/1612.01507

[12] Yin Tat Lee and Santosh S. Vempala. 2016. Geodesic Walks in Polytopes. CoRR abs/1606.04696 (2016). http://arxiv.org/abs/1606.04696

[13] Yin Tat Lee and Santosh Srinivas Vempala. 2017. Eldan's Stochastic Localization and the KLS Hyperplane Conjecture: An Improved Lower Bound for Expansion.
In Proc. of IEEE FOCS.

[14] L. Lovász. 1990. How to compute the volume? fber. d. Dt. Math.-Verein, fubiläumstagung 1990 (1990), 138-151.

[15] L. Lovász and M. Simonovits. 1990. Mixing rate of Markov chains, an isoperimetric inequality, and computing the volume. In ROCS. 482-491.

[16] L. Lovász and M. Simonovits. 1993. Random walks in a convex body and an improved volume algorithm. In Random Structures and Alg., Vol. 4. 359-412.

[17] L. Lovász and S. Vempala. 2006. Fast Algorithms for Logconcave Functions: sampling, Rounding, Integration and Optimization. In FOCS. 57-68.

[18] L. Lovász and S. Vempala. 2006. Hit-and-run from a corner. SIAM f. Computing 35 (2006), 985-1005. Issue 4.

[19] L. Lovász and S. Vempala. 2006. Simulated annealing in convex bodies and an $O^{*}\left(n^{4}\right)$ volume algorithm. F. Comput. Syst. Sci. 72, 2 (2006), 392-417.

[20] L. Lovász and S. Vempala. 2007. The geometry of logconcave functions and sampling algorithms. Random Struct. Algorithms 30, 3 (2007), 307-358.

[21] Oren Mangoubi and Aaron Smith. 2017. Rapid Mixing of Hamiltonian Monte Carlo on Strongly Log-Concave Distributions. arXiv preprint arXiv:1708.07114 (2017).

[22] R.M. Neal. 1996. Bayesian Learning for Neural Networks. Springer New York.

[23] Radford M. Neal. 2010. MCMC Using Hamiltonian Dynamics. Handbook of Markov Chain Monte Carlo 54 (2010), 113-162. 